\title{
Philosophiques
}

\section{Propriété et détermination : Sémantique et ontologie chez Bernard Bolzano}

\section{Jocelyn Benoist}

Volume 30, numéro 1, printemps 2003

Bernard Bolzano. Philosophie de la logique et théorie de la connaissance

URI : https://id.erudit.org/iderudit/007736ar

DOI : https://doi.org/10.7202/007736ar

Aller au sommaire du numéro

Éditeur(s)

Société de philosophie du Québec

ISSN

0316-2923 (imprimé)

1492-1391 (numérique)

Découvrir la revue

Citer cet article

Benoist, J. (2003). Propriété et détermination : Sémantique et ontologie chez Bernard Bolzano. Philosophiques, 30(1), 137-148.

https://doi.org/10.7202/007736ar
Résumé de l'article

L'auteur essaie de circonscrire la sphère du " métaphysique " dans la pensée de Bolzano. Il montre comment la métaphysique de la réalité (Wirklichkeit), avec ses deux ingrédients : les substances et les propriétés (Beschaffenheiten), doit être distinguée de la doctrine du règne " sémantique " (celui des représentations et des propositions en soi). Ces dernières entités n'appartiennent pas à la sphère de l'ontologie, et il est impossible de trouver quelque chose comme une " ontologie formelle » chez Bolzano qui serait en charge de s'occuper d'elles, c'est-à-dire une doctrine qui les traiterait comme des « êtres ". L'auteur s'intéresse, de ce point de vue, à la distinction importante faite par Bolzano, entre les propriétés (Beschaffenheiten), qui doivent être prises en un sens ontologique, et les déterminations (Bestimmungen) qui, bien que rapportées à des objets et éventuellement des êtres, n'ont pas de sens indépendamment d'un discours tenu sur ces objets, et ne sont pas des entités ontologiques à proprement parler. Ainsi, l'auteur essaie de mettre en lumière la complexité des relations entre le plan sémantique et le plan ontologique chez Bolzano : les deux plans doivent être soigneusement distingués, et pourtant demeurent aussi corrélés en un sens complexe.
Ce document est protégé par la loi sur le droit d'auteur. L'utilisation des services d’Érudit (y compris la reproduction) est assujettie à sa politique d'utilisation que vous pouvez consulter en ligne.

https://apropos.erudit.org/fr/usagers/politique-dutilisation/ 


\title{
Propriété et détermination : Sémantique et ontologie chez Bernard Bolzano
}

\author{
JOCELYN BENOIST \\ jocelyn.benoist@noos.fr \\ Université de Paris I/Archives-Husserl
}

\begin{abstract}
RÉSUMÉ. - L'auteur essaie de circonscrire la sphère du «métaphysique » dans la pensée de Bolzano. Il montre comment la métaphysique de la réalité (Wirklichkeit), avec ses deux ingrédients : les substances et les propriétés (Beschaffenheiten), doit être distinguée de la doctrine du règne «sémantique» (celui des représentations et des propositions en soi). Ces dernières entités n'appartiennent pas à la sphère de l'ontologie, et il est impossible de trouver quelque chose comme une «ontologie formelle» chez Bolzano qui serait en charge de s'occuper d'elles, c'est-à-dire une doctrine qui les traiterait comme des «êtres ». L'auteur s'intéresse, de ce point de vue, à la distinction importante faite par Bolzano, entre les propriétés (Beschaffenheiten), qui doivent être prises en un sens ontologique, et les déterminations (Bestimmungen) qui, bien que rapportées à des objets et éventuellement des êtres, n'ont pas de sens indépendamment d'un discours tenu sur ces objets, et ne sont pas des entités ontologiques à proprement parler. Ainsi, l'auteur essaie de mettre en lumière la complexité des relations entre le plan sémantique et le plan ontologique chez Bolzano : les deux plans doivent être soigneusement distingués, et pourtant demeurent aussi corrélés en un sens complexe.
\end{abstract}

\begin{abstract}
The author tries to define the realm of the "metaphysical" in Bolzano's work. He shows how the metaphysics of reality (Wirklichkeit), with both its ingredients : substances and proprieties (Beschaffenheiten), has to be contrasted with the doctrine of the semantic realm (the realm of representations and propositions in themselves). The latter do not belong to ontology, and it is impossible to find something like a "formal ontology" dealing with them in Bolzano's work, viz. a doctrine describing them as "beings". The author deals, from this point of view, with the important distinction made by Bolzano, between proprieties (Beschaffenheiten), that have to be taken in an ontological sense, and determinations (Bestimmungen), that, though related to objects and even beings, do not make sense independently from the existence of some discourse on those objects, and are not ontological entities properly speaking. Thus, the author attempts to highlight the complexity of the relations between the semantic and the ontological level in Bolzano's work : both have to be distinguished accurately, and however remain correlated in a complex way.
\end{abstract}

Chez Bolzano, conformément à la tradition aristotélicienne dont il est issu, on trouve conjugués un projet de remise en forme de la logique, qu'il accomplit dans la Wissenschaftslehre, et un point de vue de métaphysicien, qui s'exprime dans d'autres textes, comme l'Athanasia, mais qui demeure aussi constamment présent dans les traités plus proprement logiques. Logique et métaphysique sont largement inséparables pour le philosophe tchèque, et la conception de la logique qu'il présente dans la Wissenschaftslehre est très

PHILOSOPHIQUES 30/1 — Printemps 2003, p. 137-148 
directement déterminée par des positions métaphysiques, et plus particulièrement ontologiques, qui y sont aussi explicitement défendues.

Cet engagement métaphysique, après avoir sans doute constitué un obstacle à la réception de Bolzano, peut expliquer le nouvel intérêt dont il semble faire l'objet alors qu'une certaine philosophie analytique déplace son attention de l'énoncé lui-même vers «ce qui le rend vrai » (les truth-makers), donc le correspondant, dans le « réel », de l'énoncé. L'analyse ne se conçoit dès lors plus sans une face proprement ontologique. Or, Bolzano, quant à lui, n'avait jamais réellement séparé logique et ontologie, et son œuvre semble nous donner d'amples ressources en matière de théorisation de ce qui «correspond " aux énoncés et ce qui les rend vrais ou non. Du point de vue qui est le sien, une bonne théorie logique ne se conçoit pas en dehors d'une prise en compte minutieuse de ce qui est, et surtout des différences dans l'ordre de ce qui est.

Pour autant, est-il possible de réduire ce qu'il fait dans la Wissenschaftslehre, et notamment dans sa partie fondamentale, l'Elementarlehre, à un propos immédiatement et directement ontologique?

Il nous semble que ce serait aller trop vite - comme le font aujourd'hui un certain nombre de lecteurs néo-ontologues de Bolzano. Trop souvent, la référence à Bolzano est convoquée aux fins de l'édification de ce qu'on appelle une «ontologie formelle», pour reprendre une expression à la mode. Si cette formule renvoie à quoi que ce soit comme une ontologie de la variable, certainement, elle est fausse - le concept de variable étant peu bolzanien, à la différence de celui de variation, mais c'est tout autre chose. Mais il ne semble pas que ce soit nécessairement ce que les tenants de l'« ontologie formelle» ont en vue. Le mérite de Bolzano, de leur point de vue, serait plutôt d'avoir enrichi notre panoplie ontologique en y introduisant de nouvelles entités, et en donnant ainsi, pour la première fois, les moyens d'une véritable ontologie formelle.

En quoi celle-ci serait-elle «formelle» ? Tout simplement, semble-t-il, en ce qu'elle comprendrait en son sein des entités «non-réelles» (formel devant ici s'opposer à réel au sens de l'effectivité). La percée ontologique de Bolzano tiendrait alors dans l'institution par lui d'entités telles que lesdites représentations et propositions «en soi».

Lire Bolzano ainsi revient évidemment à le tenir pour un platoniste quelle que soit la façon qu'on ait, après coup, de monnayer ce platonisme, suivant le degré d'attraction ou de répulsion que l'on a soi-même pour ce genre de position.

Or, il nous semble que ce genre de lecture rencontre de grandes difficultés. La principale est l'impossibilité dans laquelle elle nous met d'apprécier la nature exacte de ce que fait le philosophe pragois dans l'Elementarlehre de la Wissenschaftslehre. Qu'il y ait beaucoup d'ontologie dans ces pages — et un poids déterminant de l'ontologie y compris sur ce qui n'est pas proprement ontologique, conformément aux vœux d'une philosophie analytique plus « ontologique» que celle des grandes années du linguistic turn — ce n'est pas douteux. Pour autant relèvent-elles vraiment de l'ontologie? Constituent- 
elles un chapitre supplémentaire ( «formel») au grand manuel de l'ameublement du monde, tel que l'ontologie traditionnelle pouvait l'entendre?

Notre hypothèse personnelle serait que les lire ainsi conduirait à passer à côté de ce qu'essaie d'y établir Bolzano, à savoir une sémantique (une doctrine du sens, qui n'est pas de l'être), qui, si elle suppose bien une ontologie, et ne peut se penser que par rapport à elle, ne se confond nullement avec elle, et ne peut être présentée sur le même plan et dans le même sens qu'elle, si ce n'est sur un mode parodique.

C'est ce que nous essaierons d'instruire ici en sondant certains des écarts explicites que Bolzano creuse entre sémantique (théorie des représentations et des propositions en soi) et ontologie, notamment celui qu'illustre, dans sa pensée, le doublage de la notion (ontologique) de propriété par celle (proprement sémantique) de détermination.

Il y a certainement une équivoque dans l'institution bolzanienne des notions de représentation et de proposition en soi. Par certains côtés, Bolzano semble emprunter une argumentation de type ontologique qui anticipe Meinong en allant à l'encontre du "préjugé en faveur de l'effectivité ». On ne peut attribuer aux propositions en soi (concept primitif, les représentations en soi ne pouvant être définies que comme des parties desdites propositions en soi) ni l'existence (Dasein ou Existenz), ni l'effectivité (Wirklichkeit) ${ }^{1}$. N'estce pas à dire qu'il faille leur reconnaître un être (Sein) qui déborde le sens de la seule effectivité?

En fait, Bolzano fait une différence qui est un peu de cet ordre, mais plutôt que de passer entre deux types d'êtres, oppose à l'être au sens rigoureux du terme (ce qui est : das Seiende) la chose (Ding), dont on n'a pas nécessairement à dire qu'elle "soit ». Pour Bolzano, les propositions en soi et leurs constituants représentationnels sont bien des choses, même s'il ne saurait être question de leur attribuer l'effectivité. Ni même, en toute rigueur, l'être. Pour le philosophe de Prague, le seul être "au sens propre du terme (eigentlich) » (il reprend ainsi une opposition aristotélicienne entre le propre et l'impropre, qui permet de distinguer ce qui est réellement "ontologique" et ce qui ne l'est pas) est en effet celui de l'effectivité. Mais, à côté du domaine des choses qui existent - et donc sont au sens seul propre du terme - il y a "l'empire des choses qui ne prétendent ni exister ni seulement être possibles $»^{2}$ (ce dernier concept modal n'ayant lui-même de sens que relativement à la possibilité d'une existence). Et ces "choses" sont constituées essentiellement par les entités de ce type que nous nommerions sémantique : propositions et représentations en soi - qui s'opposent à la réalité effective des représentations effectivement pensées et des propositions effectivement énoncées ou proférées, sans pour autant former une autre sphère d'être («idéal»).

1. Bolzano, Bernard, Wissenschaftslehre, Sulzbach, 1837, $\mathbb{\$} 19$, vol. 1, p. 78.

2. Bolzano, Bernard, Les Paradoxes de l'infini, trad. fr. Hourya Sinaceur, Paris, Seuil, 1993, $\mathbb{1 3}$, p. 71. 
C'est dire que prima facie, l'univers de Bolzano, à défaut de son « ontologie» stricto sensu, est un univers riche, plus riche certainement que ne pourraient le supporter bon nombre de philosophies, à tendance nominaliste. Bolzano construit un sens général de la "chose ", qui inclut, à côté des choses réelles (essentiellement les substances, ou ce qui dépend des substances, nous allons y revenir), les entités de type sémantique, d'un côté ce qui a droit au qualificatif d'étant, et de l'autre ce qui n'y a pas droit : «il n'y a que deux genres de choses : les étants et celles qui ne sont pas, par exemple les substances et les vérités en soi ${ }^{3}$. Sans que cela prenne exactement le même sens que l'extra-être (Aussersein) meinongien, on semble bien avoir affaire, ici aussi, à une forme de débordement de l'ontologie qui en est une généralisation, l'ensemble des entités étant rangées sous cette forme générale qui est celle de la «chose».

Cette dimension d'ontologie généralisée (par là-même, peut-être, $\mathrm{d}$ ' "ontologie formelle») est particulièrement nette lorsque Bolzano, dans la Wissenschaftslehre, introduit le concept de «représentation en soi » — qui est essentiellement une partie de proposition en soi non porteuse en elle-même d'une valeur de vérité. Le philosophe pragois parle alors, à propos de la «représentation en soi ", de ce "quelque chose (Etwas) qu'il ne faut pas chercher dans le domaine de la réalité effective». Cette représentation, qui en elle-même (dans son statut d' «en-soi ») n'a pas besoin de sujet qui la représente, "subsiste (besteht) », dit-il, «cela assurément pas comme quelque chose d'étant (etwas Seiendes), mais pourtant comme un certain quelque chose (Etwas) ${ }^{4}$. Le quelque chose — qui toutefois, Bolzano est clair là-dessus, ne saurait être mesuré a priori par une "pensabilité » quelconque ${ }^{5}$ - semble constituer ici le genre suprême qui vient en quelque sorte se substituer à l'être : dans la sphère du quelque chose, il faudra distinguer entre ce qui a sens d'être, c'est-à-dire d'effectivité, et ce qui n'a radicalement pas un tel sens — et demeure bien pourtant un quelque chose, n'est pas «rien », au point que cela participe de plein droit à la problématique bolzanienne du « «il y a », qui s'applique aussi bien aux représentations et propositions en soi qu'aux réalités effectives. Un type d'entités comme l'autre peuvent, en effet, faire l'objet de jugements existentiels, et relèvent donc bien de ce qu'on serait tenté d'appeler - en utilisant toutefois des termes non bolzaniens - une forme $\mathrm{d}^{\prime}$ ' existence logique ${ }^{6}$.

On voit bien le bénéfice que peuvent espérer tirer de cette problématique du «quelque chose en général », qui s'applique aussi aux entités sémantiques, les tenants contemporains d'une «ontologie formelle»: l'élargissement et la

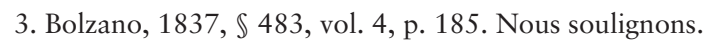

4. Bolzano, 1837, $\mathbb{S} 48$, Bd. I, p. 217.

5. Cf. le $\mathbb{S} 99$ de la Wissenschaftslehre, que nous avons étudié en détail dans «Pourquoi il n'y a pas d'ontologie formelle chez Bolzano ", Les Études philosophiques, 2000, p. 505-518. 6. Cf. Sebestik, Jan, Logique et mathématique chez Bernard Bolzano, Paris, Vrin, 1992, p. 132-133. 
diversification de nos concepts ontologiques, en dehors des seules limites de la « «réalité » effective. Cette ouverture serait marquée, chez Bolzano, par l'apparition fugitive, et apparemment distinctive (contre le Sein de ce qui est "réellement»), au $\mathbb{} 48$ de la Wissenschaftslehre, du verbe bestehen, traditionnellement convoqué par les philosophes de langue allemande là où ils essaient de dire quelque chose qui n'est plus vraiment de l'ordre d'un être ou d'une existence au sens habituel (substantiel ou para-substantiel) du terme ${ }^{7}$.

Cette présentation, qui peut donc s'appuyer sur certains énoncés ou certains emplois bolzaniens, a cependant l'inconvénient de mettre sur le même plan — aux fins de l'édification d'une espèce d' "ontologie généralisée » précisément - ce qui, semble-t-il, gagnerait à être distingué afin de mieux saisir l'intention de la problématique bolzanienne.

En fait, il faut remarquer que nulle part Bolzano ne parle d'une telle ontologie générale, ni même, en se rappelant que l'être, pour lui, ne peut recouvrir que la moitié — «réelle» — de la sphère des «choses», d'une théorie systématique des «choses » qui tienne lieu, alors, d'un substitut plus général à l'ontologie. Découvrir qu'il y a des choses qui ne «sont» pas à proprement parler, plutôt que jeter les bases d'une théorie générale du quelque chose, qui relaie l'ontologie au-delà d'elle-même et ressaisisse tout dans un ensemble unifié (celui des «choses» en tant que purs «quelque chose»), c'est mesurer l'incommensurabilité de deux genres, et s'installer dans un écart essentiel.

Aussi, partout où Bolzano fait œuvre de métaphysicien et d'ontologue (comme c'est le cas au début de l'Athanasia), reste-t-il sur le terrain du réeleffectif, seule forme possible, selon lui, de l'être, et l'extra-être du purement «subsistant»(bestehend), n'est-il jamais considéré par lui comme le complémentaire de la Wirklichkeit, contribuant, au même titre qu'elle ou au-delà d'elle, à l'ameublement du monde. Le monde, fondamentalement, c'est le monde réel, avec ses différents constituants, physiques ou métaphysiques.

Nulle part l'ontologie à proprement parler de Bolzano n'est mieux exposée qu'au début de l'Athanasia, c'est-à-dire certes avant la Wissenschaftslebre, mais d'une façon qui, croyons-nous, n'est pas fondamentalement remise en question par elle (à preuve, la seconde édition de 1838, donc après la Wissenschaftslehre, non modifiée sur ce point) :

Tout ce qui est, c'est-à-dire qui subsiste dans la réalité effective, qui subsiste dans cette réalité effective soit pour toujours soit ne serait-ce que pour un certain temps, relève de l'un ou l'autre des genres suivants : soit cela est et subsiste sur quelque chose d'autre, comme propriété de cette chose, soit ce n'est pas une simple propriété sur quelque chose d'autre, mais cela subsiste, comme on a coutume de le dire, pour soi. La couleur, l'odeur, le poids d'un corps nous fournis-

7. Cet emploi sera moqué par Brentano, qui souligne comment il est, en allemand courant, difficile de distinguer aussi clairement entre les différents verbes ontologiques ou para-ontologiques, dans la diversité de leurs emplois coutumiers et tout sauf ordonnés (Kategorienlehre, dictée du 22 mars 1916, dir. Alfred Kastil, Hamburg, Felix Meiner, 1933, p. 30-31). 
sent des exemples du premier genre; car toutes ces choses sont quelque chose de réel-effectif, qui ne subsiste pourtant pas pour soi, mais seulement sur quelque chose d'autre, à savoir en l'occurrence le corps, et alors assurément comme propriété de celui-ci. Un exemple du second genre est donné avec la matière, dont le corps est composé; car cette matière est quelque chose de réel-effectif, qui ne subsiste sur aucun autre réel-effectif comme propriété, mais le fait déjà pour soi. Les réalités effectives du premier genre, les philosophes ont coutume de les appeler d'un mot latin adhérences, celles du dernier genre substances ${ }^{8}$.

«Tout ce qui est, c'est-à-dire qui subsiste dans la réalité effective»: on ne peut pas être plus clair, c'est bien la réalité effective (Wirklichkeit) qui donne la mesure de l'être (alles, was ist). D'autre part, on notera que la notion de subsistance (par quoi nous rendons ici bestehen) ne peut manifestement être si nettement opposée à celle d'existence (être dans l'ordre de la Wirklichkeit) que nous l'avons suggéré en commentant le fameux passage du $\mathbb{} 48$ de la Wissenschaftslehre sur la subsistance des représentations en soi, qui ne "sont " pas. S'il y a des choses qui "subsistent» sans être, il faudra bien dire des choses qui existent qu'elles "subsistent" dans la mesure même où elles existent, et qu'en elles subsistance et existence se confondent. C'est tout au moins le sens pris par la notion de bestehen dans le passage de l'Athanasia que nous citons : la notion de subsistance, quelles que soient les ressources qu'y découvrira par après Bolzano dans la Wissenschaftslehre, n'apparaît ici (c'est-à-dire dans une analyse proprement ontologique, qui est nécessairement celle de la réalité effective) guère que comme un redoublement de celle d'existence - cela d'ailleurs conformément au génie le plus ordinaire de l'allemand. Sur ce terrain, proprement ontologique, il ne saurait être question d'une autre existence qu'effective.

L'important, pour Bolzano, est, dans ce domaine de la réalité effective, de distinguer deux types de réalités : celles qui sont autonomes et supportent ontologiquement ce monde, les substances; et celles de ce qui n'a de réalité que dépendante des premières réalités, à savoir l'ensemble des propriétés (Beschaffenheiten) desdites substances, propriétés que Bolzano appelle aussi «adhérences ", pour marquer leur dépendance ontologique. L'être du deuxième type de réalité n'est pour ainsi dire que second. Ce n'en est pas moins de l'être. Il y a ce qui est "pour soi » (für sich), a le statut d' "être séparé », comme dirait Aristote, et il y a ce qui ne peut être que sur (an) quelque chose d'autre - et donc suppose l'être autonome, de premier type, comme sa condition préalable.

Un monde de substances et de propriétés qui sont «sur» des substances, en dépendance d'elles et relativement à elles : peut-on rêver un monde plus explicitement aristotélicien? Or, on a là le fond de l'ontologie à proprement parler de Bolzano, explicite encore dans la Wissenschaftslehre et constamment sous-jacente aux analyses plus sémantiques qui y sont menées : d'un côté les substances, qui constituent le fond même des «choses» au sens cette

8. Bolzano, Bernard, Athanasia oder Gründe für die Unsterblichkeit der Seele (2 éd.) Sulzbach, 1838 (Frankfurt am Main, Minerva Verlag, 1970), p. 21. 
fois familier du terme (qu'il soit physique, comme dans le cas de l'objet perceptif, ou métaphysique, comme dans le cas de l'âme, qui est une substance immatérielle), et de l'autre leurs propriétés, leurs adhérences. L'univers de Bolzano, qui constitue le soubassement de son ontologie, est fondamentalement un univers de "choses", au sens le plus commun et aristotélisant du terme, c'est-à-dire de substances et de ce qui en dépend. Les "choses" formelles, au sens où la "chose » est mise à la hauteur du «quelque chose » dans sa nudité (en dehors du fait pour elles d'être des substances ou quoi que ce soit de la substance), n'y ont pas vraiment droit de cité : elles relèvent d'une autre problématique, non plus ontologique, mais sémantique.

Et en effet, l'un des aspects les plus intéressants de la Wissenschaftslehre est la façon dont Bolzano, partant d'une ontologie strictement et robustement substantialiste, creuse toute une série d'écarts entre l'ordre de l'être, c'est-à-dire du réel, avec sa structuration propre (conçue dans les termes : substance-adhérences) et celui de la représentation, qui le reflète, mais fort imparfaitement, et suivant une logique qui lui est propre, et ne peut nullement être tenue pour une simple réplique de la structure ontologique.

Que l'ordre des représentations ne soit pas un simple reflet de celui des choses, contrairement à ce que croyait Leibniz, c'est un fait déterminant pour l'ensemble de la Wissenschaftslehre ${ }^{9}$, et nous n'y reviendrons pas ici. La composition de la représentation a sa méréologie, qui n'est pas un décalque de la structure méréologique du réel (la notion même d'adhérence suggérant bien quelque chose comme une méréologie dans le réel lui-même), et au fond la Wissenschaftslehre (tout au moins l'Elementarlehre) n'a d'autre sens que d'explorer cet écart, en tentant de reconstruire les connexions qui existent entre ces deux plans (celui de la représentation et celui de l'être), dans leur dissymétrie même. Si l'ordre des représentations est bien en un sens une image de celui du réel, ou en tout cas peut l'être, c'en est une image indirecte, et il importe de retracer les voies (de composition interne) par lesquelles les représentations, assemblées d'une certaine façon, parviennent à exprimer quelque chose de ce qui est.

Mais sans doute le plus intéressant, la pointe du dispositif, est lorsque, dans une sorte d'effet de retour de la sémantique sur l'ontologie, de la représentation sur l'être, certaines distinctions apparaissent pourtant sur le second terrain ( «ontologique ») qui n'ont de sens qu'en vertu de structures qui relèvent du premier (donc de l'ordre des représentations elles-mêmes). On touche alors l'aspect le plus novateur peut-être de la pensée de Bolzano dans la Wissenschaftslehre, qui est, dans la distinction ferme même qui est maintenue de l'ontologique au sémantique, dans leur indépendance relative, la reconnaissance d'effets de reports de l'un sur l'autre. Il n'y a pas de fécondité ontologique propre du sémantique (ce n'est jamais le fait d'avoir été visée par une

9. Cf. Bolzano, 1837, $\mathbb{\$}$ \$63-64, et notre commentaire détaillé là-dessus dans «Bolzano, Husserl et l'idée de grammaire ", Les Études philosophiques, 1999, p. 521-534. 
représentation qui crée une substance, et rien n'est plus éloigné de Bolzano que quelque perspective intentionaliste que ce soit en un sens constitutif, dans la mesure où il ne peut de toute façon y avoir, dans l'ordre de l'être, que des substances et les propriétés qui en dépendent, des adhérences), mais pourtant tout se passe comme s'il y avait des effets pseudo-ontologiques du sémantique. Quelque chose comme le reflet de la représentation sur l'être - ce qui ne veut pas dire que la représentation constitue pour autant quoi que ce soit de l'être, mais cela peut être un principe (relatif) d'organisation de l'être que d'être représenté.

C'est ce qu'illustre la différence, fondamentale, faite par Bolzano entre propriété (Beschaffenheit) et détermination (Bestimmung), différence qui marque une fois de plus la radicalité de l'écart qu'il y a, dans la pensée du philosophe pragois, entre sémantique et ontologie (puisqu'à la notion directement ontologique de propriété s'oppose celle, seulement sémantique, de détermination), mais met aussi en relief la réelle complexité du rapport d'un plan avec l'autre, puisqu'ici est envisagée entre eux une véritable circulation.

Un passage des Paradoxes de l'infini nous permettra d'apprécier le problème :

Temps et espace sont une espèce très importante de grandeurs infiniment grandes qui, tout en étant des déterminations dans le réel, n'appartiennent pourtant pas à son domaine. Ni le temps ni l'espace ne sont quelque chose de réel; car ils ne sont ni des substances ni des propriétés de substances; ils entrent en jeu seulement comme déterminations de toutes les substances imparfaites (limitées, finies, ou, ce qui revient au même, dépendantes, créées), du fait que chacune d'entre elles se trouve toujours en un temps et en un lieu déterminés ${ }^{10}$.

On a ici le paradoxe de notions - à propos desquelles précisément Bolzano est amené, dans la Wissenschaftslehre, à introduire le concept de détermination - qui, tout en ne renvoyant elles-mêmes à rien de réel, n'ont de sens qu'appliquées à du réel. Le temps et l'espace ne sont rien de réels c'est-à-dire ni des substances, ni même des propriétés de substance au sens propre du terme (ce qui serait encore leur conférer une forme de consistance ontologique). Mais d'un autre côté, "déterminations", ils le sont bien de choses réelles : des réalités finies en tant que telles.

Mais qu'est-ce alors qu'une "détermination ", si ce n'est pas une propriété, donc une dimension de l'être même, mais quelque chose de l'être (au sens de : à propos de l'être) qui est installé sur un autre terrain que l'être même (celui de la représentation)? Le risque est alors grand, en retour du concept «subjectif» de représentation (la représentation comme "représentation pensée »), de faire de ces «déterminations » des propriétés non plus de ce qui est, mais de ce qui est pensé — donc purement "subjectives". C'est pourtant une éventualité repoussée avec la dernière énergie par Bolzano : le 
temps et l'espace ne sont certes rien de réel, mais «il est encore plus absurde de s'imaginer qu'[ils] ne recoivent leur réalité que de notre pensée » ${ }^{11}$. Les propriétés d'espace et de temps ne dépendent certainement pas «de notre pensée ou de notre croyance». Elles sont objectivement attachées à tel ou tel être - en toute indépendance, suivant le réalisme bolzanien, de cet être par rapport aux conditions de notre pensée.

La remarque bolzanienne est, du point de vue d'une analyse "ordinaire » de nos énoncés comportant des déterminations de temps ${ }^{12}$ ou d'espace, tout à fait pertinente : il est bien vrai que la détermination de temps (ou d'espace) est toujours présentée comme une détermination objective, attachée à la chose même, donc à de l'être - ce qui est dans le temps et/ou dans l'espace étant, par construction, un étant —, quelles que soient du reste les difficultés que nous ayons à accéder à une telle détermination et les incertitudes qui planent sur elle.

Mais alors, que sont ces «déterminations » qui flottent en quelque sorte dans l'objectivité de l' «en-soi » bolzanien, qui est un en-soi sémantique, et qui, par là-même ne sont pas de l'être, et pourtant (c'est leur caractéristique, qui les isole dans l'ordre de la représentation), disent quelque chose de l'être? L'objectivité d'une propriété est, d'un point de vue métaphysique "réaliste », chose relativement aisée à comprendre. L'objectivité d'une détermination au sens de Bolzano est moins évidente, cela dans la mesure même où, loin d'être simplement une objectivité sémantique comme une autre (comme celle de n'importe quelle représentation, sise dans cet en-soi, qui, pour Bolzano, est celui du sens), elle demeure bien plus que cela : objectivité par rapport à un être, sans être vraiment non plus celle d'un être — comme le serait celle d'une propriété.

Il y va là d'un entrelacs du sémantique et de l'ontologique, d'une indexation d'un plan à un autre, particulièrement fascinants. Au $\mathbb{S} 80$ de la Wissenschaftslehre, précisément dans la foulée de l'élucidation des concepts de temps et d'espace, Bolzano précise la différence entre propriété et détermination. "Tout ce qui échoit (zukommt) à un objet, que cela soit durablement, ou en un temps quelconque, fût-il bref, même en un seul instant, est pour ce point temporel même une propriété (Beschaffenheit) de l'objet. " ${ }^{13}$ La notion de propriété est donc clairement ontologique, comme le marquait déjà le début de l'Athanasia, en faisant la seconde catégorie ontologique essentielle - après celle de substance. C'est l'objet même qui porte ses propriétés, fussent-elles épisodiques, et elles-mêmes constituent bien un fragment - dépendant, mais légitime dans son ordre propre, qui n'est pas «rien» — de réel.

Aux propriétés correspondent, du côté sémantique, les représentations de propriétés (Beschaffenheitsvorstellungen). Celles-ci ont pour caractéris-

11. Op.cit., p. 81.

12. Cf. Bolzano, 1837, $\mathbb{} 185$ : Sätze, die Zeitbestimmungen enthalten.

13. Bolzano, 1837, $\mathbb{8} 80$, vol. 1, p. 379 . 
tique de se laisser inclure, selon la forme canonique de l'énoncé selon Bolzano ('A a $b$ ', avec A terme concret et $\mathrm{b}$ terme abstrait) dans un énoncé à la place du prédicat (celle de $b$ ). Cette aptitude constitutive des représentations de propriétés à jouer le rôle de prédicats en fait, dit Bolzano, de plein droit des déterminations qui déterminent l'objet représenté, selon la forme canonique de l'énoncé, par la représentation-sujet A.

On remarquera ici que la notion de détermination ne s'applique pas à la propriété elle-même, mais à la représentation de la propriété - c'est donc bien (contrairement à la notion de propriété elle-même) un concept sémantique au sens où nous l'avons construit. D'un autre côté, cette notion n'a pourtant de sens que pour autant que la représentation en question est rapportée à un objet, et dans son rapport même à cet objet : une représentation est détermination pour autant qu'elle est détermination d'un objet. Mais ce rapport qu'elle a à son objet ne se découvre ici qu'au prisme de la syntaxe qui est en propre celle de la représentation (de la "proposition en soi », mise sous sa forme canonique). Comment en effet la représentation de propriété $b$ se manifeste-t-elle comme détermination d'un objet si ce n'est en vertu du lien prédicatif qui l'unit à une certaine représentation (sujet) qui a elle-même un objet ? Et ce sens-là de la détermination, dans sa portée ontologique même elle qualifie alors des objets - se voit étroitement lié à l'opération proprement logique qu'est la prédication, par laquelle seulement il apparaît.

La limite du cas que nous venons d'envisager est que le fondement même de la détermination (la raison pour laquelle telle représentation détermine bien tel objet) paraît encore ontologique, puisqu'il s'agit de représentations de propriétés. $b$ détermine alors l'objet de A parce qu'elle-même représente quelque chose qui appartient réellement à cet objet, à savoir l'une de ses propriétés.

Est-ce toujours le cas? Bolzano envisage une généralisation de la notion de détermination au delà de celle de représentation de propriété - généralisation qui lui permet notamment de donner un statut à des irrealia comme l'espace et le temps, mais alors un statut non purement ontologique, à la frontière de la sémantique et de l'ontologie. Toute détermination n'entre pas dans la forme du $b$ de l'énoncé canonique ' $A$ a $b$ ', qui est nécessairement abstrait, donc une représentation de propriété. Il y a aussi des déterminations qui se caractérisent par leur incapacité à jouer un tel rôle prédicatif - cela n'aurait pas de sens que les prédiquer.

Telles sont les déterminations d'espace et de temps (seul mode d' 'existence» de tels irrealia, qui n'ont sens que de s'appliquer précisément comme déterminations : il n'y a pas d'espace et de temps en dehors des déterminations d'espace et de temps). Ces représentations ne peuvent à proprement parler jouer le rôle de prédicats. En revanche, elles entrent dans la composition de la représentation-sujet, et déterminent ainsi l'objet qui est représenté par elle, à telle enseigne que le prédicat ne s'applique dès lors plus à l'objet lui-même, purement et simplement, en dehors de tout espace ou tout temps, mais à l'objet tel qu'il est représenté, déterminé comme objet $\mathrm{X}$ en tel espace ou en tel temps. $\mathrm{La}$ 
notion de détermination prend ici sa véritable portée, non directement ontologique, qui est de préciser les conditions sous lesquelles quelque chose peut être dit avec vérité d'un objet.

Une détermination, dans toute la généralité du concept, est donc une représentation qui représente (aussi) l'objet d'une autre représentation. On relèvera d'ores et déjà l'ambiguité d'un concept qui, tout en ayant bien en un sens une portée ou tout au moins un champ d'application ontologique (une détermination, c'est une détermination de choses, ou tout au moins d'objets), ne la gagne par un détour via une autre représentation - la détermination n'est détermination que du fait que le même objet est aussi représenté par une autre représentation, et fait donc appel à ce fait, non ontologique, pour l'objet d'être représenté.

A partir de là, deux cas de figure sont possibles. Soit la détermination n'a d'autre sens que de capter une propriété de l'objet qui est par ailleurs déjà complètement - c'est-à-dire de façon satisfaisante gnoséologiquement, de telle façon que cela ait un sens de lui attribuer de telles propriétés - représenté. Dans ce cas-là, la logique de la détermination ne fait que déployer, dans l'ordre propre de la représentation, considéré dans son rapport avec ses objets, l'articulation ontologique même qui est celle des substances avec leurs propriétés. Soit la détermination vient modifier la représentation sujet de telle sorte que l'objet dont la propriété est énoncée dans la forme canonique ne soit pas celui qui serait représenté par sa simple représentation, mais celui qui l'est par cette représentation assortie précisément d'une telle détermination. Le nombre de fleurs qui fleurissent tout court, cela ne veut rien dire. Mais il y a un nombre de fleurs qui fleurissent aujourd'hui dans le monde ${ }^{14}$. Les représentations « aujourd'hui » et «dans le monde » sont ici des déterminations (temporelle et spatiale) qui viennent modifier et déterminer l'objet représenté par la représentation totale dans laquelle elles entrent en composition - le rapport de détermination joue ici de la partie au tout, et non de prédicat à sujet. Ces représentations n'ont en elles-mêmes aucune portée ontologique (elles ne représentent "rien ", au sens d'aucun objet), mais elles contribuent de façon décisive à préciser l'extension d'une autre représentation qui, sous cette condition, en a une - et qui, en dehors de leur travail déterminant, précisément, n'en aurait pas.

Ainsi, certaines déterminations ne font qu'enregistrer et marquer dans l'ordre sémantique un ordre ontologique préconstitué. D'autres ajustent carrément la prise du sémantique sur l'ontologique, en réglant le scope des représentations à portée ontologique en tant que celles-ci expriment l'être - alors que les représentations qui accomplissent cette fonction de réglage n'ont ellesmêmes aucune portée ontologique.

De ce point de vue, le cas de ces représentations remarquables que sont l'espace et le temps, auxquelles Bolzano, en débat contre l'Esthétique kantienne, consacre de longues analyses, est particulièrement intéressant. Pures détermi-

14. Voir la notion de truth-completer, introduite par Mark Textor dans ce contexte. 
nations — c'est-à-dire qui ne représentent aucune propriété — elles témoignent de l'écart fondamental qu'il y a, dans la pensée de Bolzano, entre le plan du sémantique et celui de l'ontologie. Dans la représentation que s'en fait Bolzano, l'espace et le temps n'ont d'autre fonction que celles de conditions de possibilité de la vérité de ce qu'on dit de certaines choses (qui ne sont telles ou telles qu'en tel ou tel temps ou tel ou tel lieu, sans que ce temps ou ce lieu puissent pour autant être tenus pour des propriétés de ces choses mêmes). En d'autres termes, on pourrait dire, en employant des termes anachroniques, que temps et espace ne sont que sur paroles. Ce sont des irrealia, qui n'ont pas vraiment d'être, et qui n'ont d'autre statut que purement sémantique, pour ainsi dire des effets de représentation: la chose n'est dans aucun espace ni aucun temps, sauf en vertu du fait d'être représentée - c'est en tant qu'on peut dire d'elle des choses qui sont vraies ou fausses qu'elle y est, cet «y être» constituant alors la mesure de ces vérités et de ces faussetés, le scope suivant lequel cela a un sens que de les évaluer. Mais par là, si déchargées ontologiquement soient-elles, ces dimensions de l'espace et du temps ont pourtant, et cela essentiellement, leur mot à dire dans ce qui peut être connu et formulé («représenté») de l'être : elles ajustent exactement les contours de l'accès (représentationnel) à lui, puisque de la vérité même, en tant que propriété fondamentalement sémantique.

Bien sûr une telle notion de "détermination » ne doit certainement pas être une fois de plus détournée en un sens subjectif : il ne s'agit pas ici de mesurer l'être à nos facultés (subjectives) de le recevoir. La détermination, fût-elle déchargée ontologiquement (comme celle d'espace ou de temps) est parfaitement objective, et en un sens « réelle» (si on entend par «réelle»: indépendante de la conscience) : elle réside dans ce qu'on aimerait appeler l'objectivité du langage — ou tout au moins du sens.

Mais en même temps cette notion nous donne un aperçu sur ce qu'est la véritable fonction de la "sémantique » chez Bolzano, une fois celle-ci désubjectivisée : celle de sas d'accès idéal, objectif à l'ontologie, cela dans sa différence même avec l'ontologie. Traiter l'une et l'autre sur un même plan (comme déploiement d'une seule et même ontologie qui comprend une partie «formelle»), aux mépris des déclarations expresses de Bolzano, reviendrait à manquer cette fonction - et sans doute par là-même ce qu'il y a de plus moderne chez le magicien de Prague. 